\title{
Numerical study of parametric pumping current in mesoscopic systems in the presence of a magnetic field
}

\author{
Fuming Xu, ${ }^{1}$ Yanxia Xing,,${ }^{1,2}$ and Jian Wang ${ }^{1, *}$ \\ ${ }^{1}$ Department of Physics and the Center of Theoretical and Computational Physics, The University of Hong Kong, Hong Kong, China \\ ${ }^{2}$ Department of Physics, Beijing Institute of Technology, Beijing 100081, China \\ (Received 28 September 2011; revised manuscript received 26 November 2011; published 28 December 2011)
}

\begin{abstract}
We numerically study the parametric pumped current when magnetic field is applied both in the adiabatic and nonadiabatic regimes. In particular, we investigate the nature of pumped current for systems with resonance as well as antiresonance. It is found that, in the adiabatic regime, the pumped current changes sign across the sharp resonance with long lifetime, while the nonadiabatic pumped current at finite frequency does not. When the lifetime of the resonant level is short, the behaviors of the adiabatic and nonadiabatic pumped currents are similar with sign changes. Our results show that, at the energy where complete transmission occurs, the adiabatic pumped current is zero, while the nonadiabatic pumped current is nonzero. Different from the resonant case, both the adiabatic and nonadiabatic pumped currents are zero at antiresonance with complete reflection. We also investigate the pumped current when the other system parameters such as magnetic field, pumped frequency, and pumping potentials are varied. Interesting behaviors are revealed. Finally, we study the symmetry relation of the pumped current for several systems with different spatial symmetries upon reversal of magnetic field. Different from the previous theoretical prediction, we find that a system with general inversion symmetry can pump out a finite current in both the adiabatic and nonadiabatic regimes with an approximate relation $I(B) \approx I(-B)$ at small magnetic field. It has been shown theoretically that for systems with reflection symmetry, the pumped current satisfies the relation $I(B)=I(-B)$ in the adiabatic regime. Our results show that even for systems evolving from the inversion to reflection symmetry, the pumped current still obeys the relation $I(B)=I(-B)$ in the adiabatic regime at small magnetic field.
\end{abstract}

DOI: $10.1103 /$ PhysRevB.84.245323

PACS number(s): 72.10.Bg, 73.23.-b, 73.40.Gk

\section{INTRODUCTION}

The idea of the parametric electron pump was first addressed by Thouless; ${ }^{1}$ it is a mechanism in which, at zero bias, a dc current is pumped out by periodically varying two or more system parameters. Over the years, there has been intensive research interest concentrated on the parametric electron pump. ${ }^{2-10}$ The electron pumping effect has been explored on a quantum-dot setup consisting of AlGaAs/GaAs heterojunction. ${ }^{11}$ Low-dimensional nanostructures, such as carbon nanotubes ${ }^{12,13}$ (CNT) and graphene, ${ }^{14,15}$ were also proposed as potential candidates. Investigation of the electron pump also triggers the proposal of spin pump, ${ }^{16-18}$ in which a spin current is induced by various means.

At the low pumping frequency limit, the variation of the system is relatively slower than the process of energy relaxation. ${ }^{19}$ Hence, the system is nearly in equilibrium and we could deal with the adiabatic pump by equilibrium methods. On the other hand, the nonadiabatic pump refers to the case wherein the pumping process is operated at a finite frequency. In the nonadiabatic regime, nonequilibrium transport theory should be employed. Theoretical methods adopted in the research field include conventional scattering matrix theory, ${ }^{5,20,21}$ Floquet scattering matrix, ${ }^{9,22}$ and the nonequilibrium Green's function (NEGF) method, ${ }^{23,24}$ as well as other methodologies to both adiabatic ${ }^{25}$ and nonadiabatic ${ }^{26}$ electron pumps.

The electron pump is a phase-coherent phenomenon since the cyclic variation of system parameters affects the phase of wave function with respect to its initial value. ${ }^{27}$ As a result, it is very sensitive to the external magnetic field. It was found theoretically, using the Floquet scattering matrix method, ${ }^{28-30}$ that the pumped current of a open quantum-dot system shows strong dependence on the spatial symmetry when the magnetic field applied in the system is reversed. Later, it was numerically suggested $^{31}$ that the pumped spin current also has certain spatial symmetries.

In this paper, we aim to numerically investigate the pumped current in the presence of magnetic field. Both adiabatic and nonadiabatic pumped currents are calculated. We focus on the nature of the pumped current for the mesoscopic systems with resonance with complete transmission and antiresonance with complete reflection. We find that the behaviors of adiabatic and nonadiabatic pumped currents are very different. Our results show that in the nonadiabatic regime, the pumped current is nonzero at resonance, while it is zero at antiresonance. However, the adiabatic pumped current is always zero regardless of types of resonance. Since there is no external driving force, the direction of current depends only on the system parameters. Our numerical results show that the adiabatic pumped current reverses its sign at the resonance or antiresonance. For the nonadiabatic pumped current, the sign reversal depends on the lifetime of the resonant states. The nonadiabatic pumped current changes the sign near the resonant point only when the lifetime is short. We also study the pumped current as a function of magnetic field. We find that as the system enters the quantum Hall regime with increasing magnetic field strength, the pumping current vanishes. Since in the quantum Hall regime the electron wave function appears as edge state, it will circumvent the confining potentials shown in Fig. 1. Pumping potentials overlapping in space with confining potentials provide no modulation on the electron wave function during 
(a) IUD

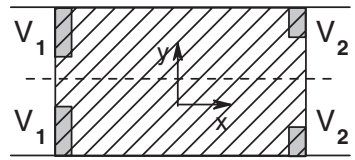

(b) ILR

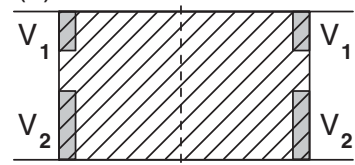

(c) IIV

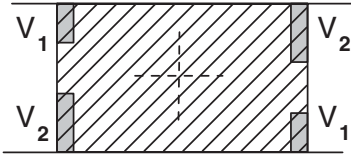

(d) GUD

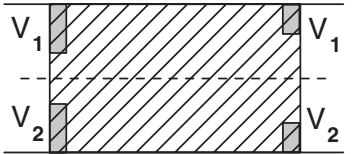

(e) GLR

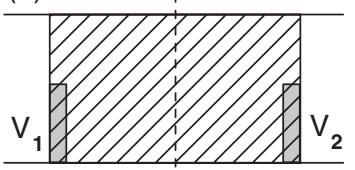

(f) GIV

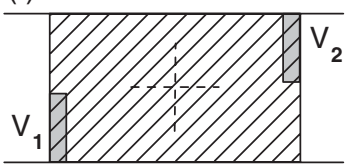

FIG. 1. Sketch of the spatial reflection symmetries: (a) instantaneous up-down (IUD); (b) instantaneous left-right (ILR); (c) instantaneous inversion (IIV); (d) general up-down (GUD); (e) general left-right (GLR); (f) general inversion (GIV). Shadow rectangules indicate the pumping region and dark gray blocks stand for potential barriers defining the spatial symmetry of the system. The pumping potentials are right on top of these confining potentials.

the variation period. Hence, there is zero pumped current in the quantum Hall regime. We also examine the pumped current and its relation with other system parameters such as pumping frequency and pumping potential amplitude. Theoretically, it was predicted that for systems that have reflection symmetry, the adiabatic pumped current remains the same when the magnetic field is reversed. Motivated by this result, we also investigate the symmetry properties of the pumped electron current of systems with certain spatial symmetries in the presence of magnetic field by the Green's function method. The electron pump is driven by periodical modulation of potentials, which share the same spatial coordinates with the confining potentials, which preserve reflection symmetry of the system.

In this paper, six spatial symmetries studied in Ref. 29 are considered, both at the adiabatic and nonadiabatic cases, which are schematically depicted in Fig. 1. For these systems, the confining potential as well as pumping potential are given in Eq. (1). Since the spatial profile of the confining potential and pumping potential are the same, the spatial symmetries are kept at any moment for the setups, Figs. 1(a)-1(c), during the pumping period. Hence, we refer to them as instantaneous up-down (IUD), instantaneous left-right (ILR), and instantaneous inversion (IIV) symmetries, respectively. On the other hand, symmetries are not preserved during the whole pumping cycle except when $\phi_{j k}=n \pi$ in setups 1(d)-1(f). They are correspondingly referred to as general up-down (GUD), general left-right (GLR), and general inversion (GIV) symmetries. In our study, all potential profiles locate at the boundary of the pumping region, i.e., the first and/or last layer in the discrete lattice (see the dark gray region).

Most of our numerical results agree with the conclusions from Floquet scattering theory ${ }^{28,29}$ except for the general inversion symmetry (GIV) [setup in Fig. 1(f)]. In contrast with the theoretical prediction that the adiabatic pumped current $I^{\text {ad }} \approx 0$ for this spatial symmetry, our numerical calculation shows that the pumped current is finite and further investigation

reveals that there is an approximate symmetry relation of the current as setup Fig. 1(e) at small magnetic field, i.e., $I(B) \approx$ $I(-B)$. Our results further show that for systems evolving from the inversion to reflection symmetry, the pumped current still obeys the relation $I(B)=I(-B)$ in the adiabatic regime at small magnetic field.

Our paper is organized as follows. In the next section, we will describe the numerical method. It is then followed by the numerical results and discussions in Sec. III. Finally, conclusions are given in Sec. IV.

\section{THEORETICAL FORMALISM AND METHODOLOGY}

We consider a quantum-dot system consisting of a coherent scattering region and two ideal leads, which connect the dot to electron reservoirs. The whole system is placed in the $x-y$ plane and a magnetic field is applied. The single electron Hamiltonian of the scattering region is simply

$$
H=\frac{(\mathbf{p}+e \mathbf{A} / c)^{2}}{2 m^{*}}+V(x, y, t),
$$

where $\mathbf{A}$ is the vector potential of the magnetic field. Here, the magnetic field is chosen to be along the $z$ direction with $\mathbf{B}=(0,0, B)$. The vector potential has only the $x$ component in the Landau gauge $\mathbf{A}=(-B y, 0,0)$. Expanding the potential term according to the time dependency

$$
\begin{aligned}
V(x, y, t) & =V_{s}(x, y)+V_{t}(x, y, t), \\
V_{s}(x, y) & =V_{0} \sum_{j} \Delta_{j}(x, y), \\
V_{t}(x, y, t) & =\sum_{j} V_{p, j} \Delta_{j}(x, y) \cos \left(\omega t+\phi_{j}\right),
\end{aligned}
$$

where $V_{s}$ is the static potential part and $V_{t}$ is the time-dependent term. $j=1$ or 2 labels the indices of the potential in our calculation setup shown in Fig. $1 . V_{0}$ and $V_{p, j}$ are amplitudes of the static potential and pumping potential, respectively. To maintain the symmetry of the system, we need to set that $V_{p, 1}=V_{p, 2}=V_{p} . \Delta_{j}$ represents the potential profile, which is a scalar vector and $\phi_{j}$ is the initial phase of the time-varying potential. Then, the Hamiltonian is expressed as

$$
H=H_{0}+V_{t},
$$

where

$$
H_{0}=\left(-i \hbar \frac{\partial}{\partial x}-\frac{e}{c} B y\right)^{2}+\left(-i \hbar \frac{\partial}{\partial y}\right)^{2}+V_{s}(x, y) .
$$

For the adiabatic electron pump, the average current flowing through lead $\alpha$ due to the slow variation of system parameter $V_{t, j}$ in one period is given by

$$
I_{\alpha}^{\mathrm{ad}}=\frac{1}{\tau} \int_{0}^{\tau} d t \frac{d Q_{\alpha}(t)}{d t}=\frac{q \omega}{2 \pi} \int_{0}^{\tau} d t \sum_{j} \frac{d N_{\alpha}}{d V_{t, j}} \frac{d V_{t, j}}{d t},
$$

where $\tau=2 \pi / \omega$ is the variation period of potential $V_{t, j}$ and $\omega$ is the corresponding pumping frequency. $\alpha=L$ or $R$ labels the lead. The so-called emissivity $d N_{\alpha} / d V_{j}$ is conventionally defined in terms of the scattering matrix $S_{\alpha \beta}$ as $^{32}$

$$
\frac{d N_{\alpha}}{d V_{j}}=\int \frac{d E}{2 \pi}\left(-\partial_{E} f\right) \sum_{\beta} \operatorname{Im} \frac{\partial S_{\alpha \beta}}{\partial V_{j}} S_{\alpha \beta}^{*} .
$$


Formally, the integration of Eq. (2) can be represented as

$$
I_{\alpha}^{\mathrm{ad}}=\frac{q}{2 \pi} \int_{0}^{2 \pi / \omega} i(\omega t) d(\omega t)=\frac{q}{2 \pi} \int_{0}^{2 \pi} i(u) d u .
$$

Clearly, the integration does not involve the frequency $\omega$ under variable substitution $u=\omega t$. For simplicity, we can always choose $\omega=1$ in the calculation, and the above expression is further simplified as

$$
I_{\alpha}^{\mathrm{ad}}=\int_{0}^{2 \pi} i^{\mathrm{ad}}(t) d t .
$$

Hence, in the calculation of $I^{\text {ad }}, V_{p}$ is the only pumping parameter and $\omega$ is absent in this regime.

In the language of Green's function, the definition of $I^{\text {ad }}$ in Eq. (2) is equivalent to the following form: ${ }^{24}$

$$
I_{\alpha}^{\mathrm{ad}}=q \int_{0}^{2 \pi} d t \int \frac{d E}{2 \pi}\left(-\partial_{E} f\right) \operatorname{Tr}\left[\Gamma_{\alpha} G^{r} \frac{d V_{t}}{d t} G^{a}\right],
$$

where the instantaneous retarded Green's function $G^{r}$ in real space is defined as

$$
G^{r}(E, t)=\left[E-H(t)-\Sigma^{r}\right]^{-1},
$$

where $\Sigma^{r}$ is the self-energy due to the leads.

For the nonadiabatic pump at finite frequency, the pumped current up to the second order in pumping potential is derived $\operatorname{as}^{23}$

$$
\begin{aligned}
I_{\alpha}^{\mathrm{nad}}= & -i q \sum_{j k=1,2} V_{p}^{2} \int \frac{d E}{8 \pi} \operatorname{Tr}\left\{\Gamma _ { \alpha } G _ { 0 } ^ { r } \Delta _ { j } \left[\left(f-f_{-}\right)\right.\right. \\
& \times\left(G_{0}^{r-}-G_{0}^{a-}\right) e^{i \phi_{k j}}+\left(f-f_{+}\right) \\
& \left.\left.\times\left(G_{0}^{r+}-G_{0}^{a+}\right) e^{-i \phi_{k j}}\right] \Delta_{k} G_{0}^{a}\right\},
\end{aligned}
$$

where $\Gamma_{\alpha}$ is the linewidth function of lead $\alpha$ defined as $\Gamma_{\alpha}=i\left[\Sigma_{\alpha}^{r}-\Sigma_{\alpha}^{a}\right] ; f=f(E)$ and $f_{ \pm}=f(E \pm \omega)$ are the Fermi distribution functions; and $\phi_{k j}=\varphi_{j}-\varphi_{k}$ is the phase difference between the two pumping potentials. Here, $G^{0 r}=$ $G^{0 r}(E)$ and $G^{0 r \pm}=G^{0 r \pm}(E \pm \omega)$ are the retarded Green's functions where there are no pumping potentials. We see from Eq. (6) that the parameter $V_{p, j}$ does not enter the calculation of the nonadiabatic pumped current, which only involves the equilibrium Hamiltonian of the system. Hence, in the calculation, it is better to evaluate the scaled current $I^{\text {nad }} / V_{p}^{2}$. Then, in the nonadiabatic regime, the pumping parameter is the frequency $\omega$ and $V_{p}$ is absent.

In the following section, we will use Eqs. (4) and (6) to carry on numerical investigations, and all the numerical work is done at the zero temperature. In the calculation, we consider a square quantum dot with size $0.7 \mu \mathrm{m} \times 0.7 \mu \mathrm{m}$. Two open leads with the same width connect the dot to the electron reservoirs. The quantum dot is then discretized into a $40 \times 40$ mesh. The hopping energy $t=\hbar^{2} / 2 m^{*} a^{2}$ sets the energy scale with $a$ the lattice spacing and $m^{*}$ the effective mass of electrons in the quantum dot. Dimensions of other relevant quantities are then fixed with respect to $t$. For instance, the unit of magnetic field strength is $13.5 \mathrm{~T}$.

\section{NUMERICAL RESULTS AND DISCUSSION}

In this section, numerical results will be presented. To test our numerical method, we first study the reflection symmetry of the pumped current on the inverse of magnetic field. Other properties of the current will be discussed in Sec. III B.

\section{A. Symmetry of pumped current}

To check the symmetry of the pumped current,, we have schematically plotted in Fig. 1 six setups with different spatial symmetries of interest. Before presenting numerical results, we would like to point out that for setup Fig. 1(c) with instantaneous inversion symmetry (IIV), the theoretical predictions $^{28,29}$ and our numerical calculations give the same result: the pumped current is exactly zero at both adiabatic and nonadiabatic cases, which is independent of $B$ and $\phi_{12}$. The phenomena can be straightforwardly understood by the Floquet scattering matrix theory. ${ }^{29}$ In the IIV setup, it is obvious that the transmission coefficient of an electron traveling from the left lead to the right $T_{R \leftarrow L}$ is always equal to that of an electron moving in the opposite direction $T_{L \leftarrow R}$, i.e.,

$$
T_{R \leftarrow L}=T_{L \leftarrow R} .
$$

From the Landauer-Büttiker formula, the electric current along the left to right region is given by

$$
I_{R \leftarrow L}=\frac{2 e}{h} \int d E T_{R \leftarrow L}(E) f(E),
$$

while $I_{L \leftarrow R}$ is defined in a similar way. Then, the pumped current through the left lead is defined as ${ }^{9,29}$

$$
I_{L}=I_{L \rightarrow R}-I_{R \rightarrow L}=0 .
$$

The conclusion holds for any particular moment, which means that there will be no pumped current at all. Hence, in the following, we will not discuss the case of setup Fig. 1(c).

First, we examine the relation between the adiabatically pumped current $I^{\text {ad }}$ and phase difference $\phi_{12}$ of the pumping potentials calculated from Eq. (4). A sinusoidal behavior is observed at a relatively small pumping amplitude $V_{p}=0.5$ for all setups in Fig. 1. The sinusoidal form of $I^{\text {ad }}\left(\phi_{12}\right)$ represents a generic property of adiabatic electron pump at small $V_{p}$. Driven by the cyclic variation of two time-dependent system parameters, the pumped current is directly related to the area enclosed by the parameters in parametric space. At small pumping amplitudes, the leading order of $I^{\text {ad }}$ is proportional to the phase difference between the pumping potentials $I^{\text {ad }} \propto V_{p} \sin \phi_{12} .{ }^{5}$ However, the relation does not hold for large pumping amplitude. To demonstrate this, we have calculated current pumping through the setup with symmetry ILR at a large potential $V_{p}=1.6$. As shown in Fig. 2, the sinusoidal relation is clearly destroyed. Except for this difference arising from the pumping amplitude $V_{p}$, there is a general antisymmetry relation between the pumped current and the phase difference $\phi_{12}$ for all setups: $I\left(\phi_{12}\right)=$ $-I\left(-\phi_{12}\right)$. Naturally, $I\left(\phi_{12}=n \pi\right)=0$. This is understandable since two simultaneously varying parameters enclose a line rather than an area in the parametric space, and the pumped current vanishes. This result, however, does not hold for the 


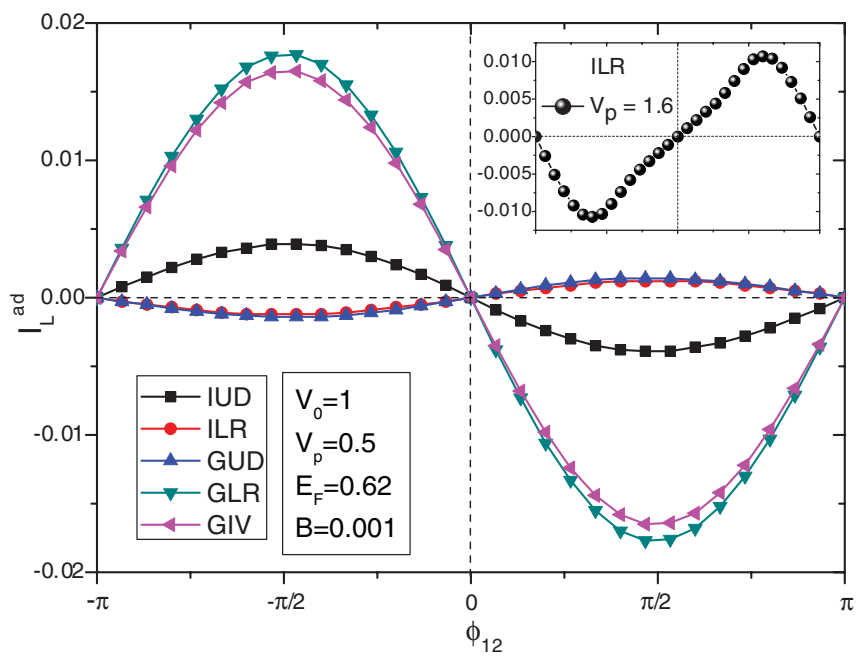

FIG. 2. (Color online) The adiabatically pumped current as a function of phase difference $\phi_{12}$ for different spatial symmetries of the pumping system at pumping amplitude $V_{p}=0.5$. Inset: pumped current of system with symmetry ILR at $V_{p}=1.6$. Other system parameters: $E_{F}=0.62, B=0.001, V_{0}=1$.

nonadiabatic case where the frequency gives an additional dimension of parametric space. Another result of interest is that, in contrast to the theoretical prediction $I^{\text {ad }} \approx 0$ for the setup of GIV symmetry, ${ }^{29}$ the pumped current from the setup of GIV is finite and has the same order of magnitude as that of GLR symmetry.

Figure 3 plots the pumped current versus magnetic field strength $B$ at phase difference $\phi_{12}=\pi / 2$, where the magnitude of $I^{\text {ad }}\left(\phi_{12}\right)$ is maximized. In Fig. 3(a), we see that the current is either an even or odd function of magnetic field strength $B, I(B)= \pm I(-B)$, for symmetries IUD, ILR, and GUD, which agrees with the theoretical predictions. ${ }^{28,29}$ In Fig. 3(b), it is clear that the pumped current is invariant upon the reversal of magnetic field for GLR. ${ }^{28,29}$ The system with GIV symmetry shows an approximation relation $I(B) \approx I(-B)$ only at small $B$, which is similar to that of GLR symmetry. This does not agree with the theoretical prediction. ${ }^{29}$ To further investigate the relation $I(B) \approx I(-B)$, we studied three intermediate setups between GIV and GLR. In Figs. 1(e) and 1(f), the length of the pumped potential profile is fixed as 20 both for $V_{1}$ and $V_{2}$ in the system with $40 \times 40$ mesh. Then, we shift down the potential profile $V_{2}$ of the GIV symmetry five lattice spacings each time. After four shifts, the system changes from GIV to GLR symmetry and in this process, three intermediate systems are generated. Numerical results shown in Fig. 3(b) suggest that all these setups have the relation $I(B) \approx I(-B)$ at small magnetic fields, although there are no spatial reflection symmetries in these systems. The closer the system is to the GLR symmetry, the larger the magnetic field for this relation. According to the unit of $B$, $B=0.0005$ in the calculation corresponds to magnetic field strength $77 \mathrm{mT}$. These results suggest that the general left-right symmetry is a rather strong spatial symmetry and even a rough setup [cyan-down-triangle curve in Fig. 3(b)] can lead to an accurate invariant relation of the pumped current, at least for small magnetic field. In addition, the amplitude of pumped

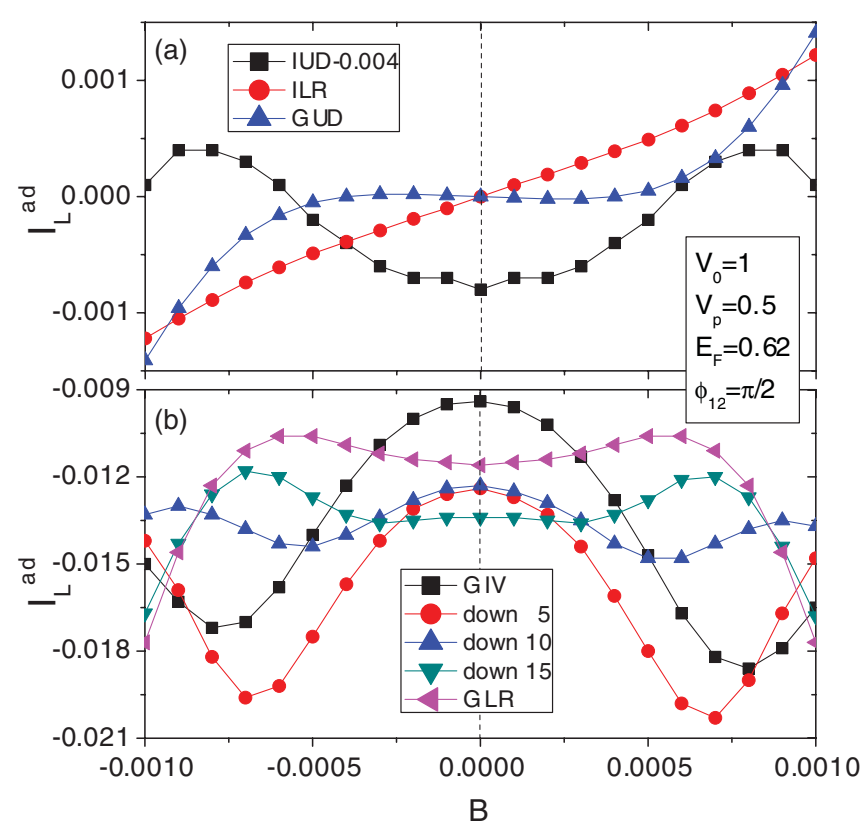

FIG. 3. (Color online) (a) The adiabatically pumped current versus magnetic field strength $B$ for system symmetries $a$ (IUD), $b$ (ILR), and $d$ (GUD). Curve for IUD is offset by -0.004 for a compact illustration. (b) $I^{\text {ad }}$ vs $B$ for spatial symmetry GLR, GIV, and three intermediate setups. Calculation parameters: $E_{F}=0.62, \phi_{12}=\pi / 2$, $V_{0}=1, V_{p}=0.5$.

current in this setup with GLR symmetry is relatively high compared with other symmetries.

Now, we turn our attention to the nonadiabatic electron pump with finite pumping frequency. The numerical results are presented in Fig. 4. One of the major differences between adiabatic and nonadiabatic pump is that a nonadiabatic pump can operate with only one system parameter since the finite pumping frequency supplies one extra degree of freedom and it could act as another pumping parameter. In the theoretical work of Ref. 23, they attributed this as a consequence of photon-assisted processes, and it is a nonlinear transport feature of nonadiabatic electron pump. In our numerical results, we also found that $I^{\text {nad }}\left(\phi_{12}=n \pi\right) \neq 0$ is a general property of the pumped current, except for systems with spatial symmetries IIV or GIV. Although the pumping frequency $\omega$ can play the role of a variation parameter, the pumped current in the system with symmetry IIV is always zero. For the setup with GIV symmetry, we see from Fig. 4(a) that the pumped current obeys an antisymmetric relation with phase difference: $I^{\mathrm{nad}}\left(\phi_{12}\right)=-I^{\mathrm{nad}}\left(-\phi_{12}\right) . I^{\mathrm{nad}}\left(\phi_{12}=n \pi\right)=0$ is a natural result of this antisymmetry relation. By combining this with the result from the adiabatic case (Fig. 2), we see that this antisymmetry relation between pumped current and phase difference $\phi_{12}$ is a general feature of the GIV symmetry. Besides, from Fig. 4(b), we found that $I^{\text {nad }}$ for the GIV system at a fixed phase $\phi_{12}=\pi / 2$ shows $I^{\mathrm{nad}}(B) \approx I^{\mathrm{nad}}(-B)$ at small magnetic field. Our results confirm the theoretical predictions on the parity of pumped current on reversal of magnetic field for setups IUD and ILR, ${ }^{29}$ which are, respectively, $I(B)=I(-B)$ and $I(B)=-I(-B)$ [see Fig. 4(b)]. However, it does not hold for GUD and GLR in Fig. 4(c). In this 

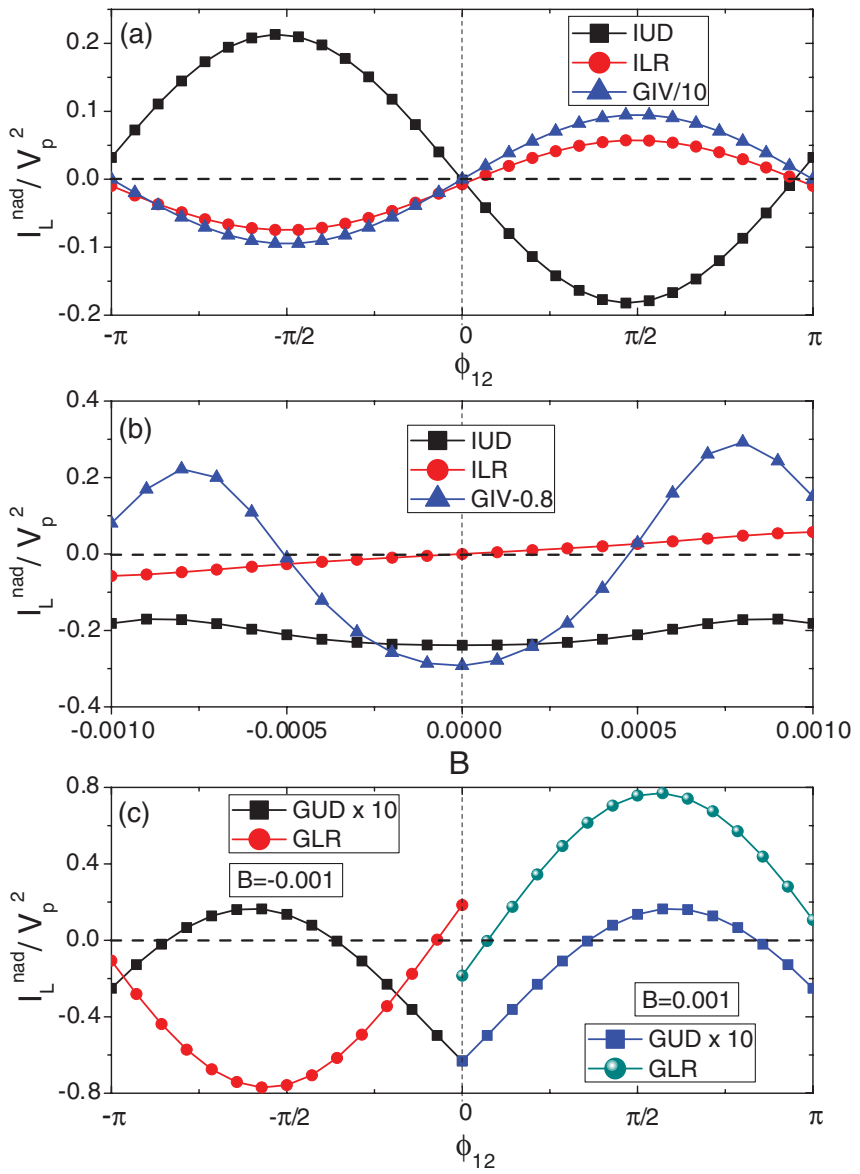

FIG. 4. (Color online) (a) Nonadiabatically pumped current as a function of phase difference $\phi_{12}$ at a fixed magnetic field $B=0.001$ for spatial symmetries IUD, ILR, and GIV. The blue curve with up-triangle for GIV is multiplied by a factor of 0.1 . (b) $I^{\text {nad }}$ versus magnetic field strength $B$ for the above three setups with $\phi_{12}=\pi / 2$. The blue curve with up-triangle for GIV is offset by -0.8 . (c) The pumped current for system setups GUD and GLR. Curves for GUD are multiplied by 0.1 . Calculation parameters: $E_{F}=0.62, V_{0}=1$, $\omega=0.002$. In all panels, a factor of 1600 is multiplied to the data for better visualization.

case, one can only get the relations $I(B, \phi)=I(-B,-\phi)$ for GUD and $I(B, \phi)=-I(-B,-\phi)$ for GLR. ${ }^{29}$ When the two pumping potentials operate in phase or out of phase $\left(\phi_{12}=n \pi\right)$, they reduce to a simple version: $I(B)=I(-B)$ for GUD and $I(B)=-I(-B)$ for GLR, which are the same for IUD and ILR at $\phi_{12}=n \pi$. It is worth mentioning that these two relations are contrary to the adiabatic case where $\phi_{12} \neq n \pi$. These conclusions drawn from both adiabatic and nonadiabatic pumps are summarized in Table. I.

\section{B. Transport properties of the pumped current}

In the preceding section, we have concentrated on the symmetry of the pumped current with magnetic field $B$ and phase difference $\phi_{12}$ as the variables. Now, we study the effect of other system parameters on the pumped current. Numerical calculations were mostly performed on a system with instant $L-R$ symmetry (ILR), in which widths of the four potential barriers are kept equal. The numerical results are plotted in the following figures.

In Fig. 5(a), we plot the pumped current in the presence of magnetic field as a function of Fermi energy $E_{F}$, together with the transmission coefficient $T\left(E_{F}\right)$ at static potential barrier $V_{0}=1.0$. The sharp tips of the transmission coefficient suggest that the quantum resonance effect dominates the transport process. When a dc bias is applied, the tunneling current is calculated from the transmission profile. However, the pumped current is generated as zero bias by periodically varying ac gate voltages. Although originating from different physical mechanisms, we see that the pumped current clearly shows resonance characteristics both in adiabatic and nonadiabatic cases near the resonant energy of the static transmission coefficient. These resonance-assisted behaviors of the pumped current are a generic property of electron pump. ${ }^{8}$ Operating at the coherent regime, quantum interference naturally results in its resonant behavior. It is worth mentioning that near the sharp resonance at $E_{F}=0.01187$, the adiabatic pumped current changes sign. This is understandable. In the presence of dc bias, the direction of the current is determined by the bias. For the parametric electron pump at zero bias, the direction of the pumped current depends only on the system parameters such as Fermi energy and magnetic field. Variation of these parameters can change the current direction. For the nonadiabatic pump, the pumped current changes slowly near the resonance, but there are no sign changes for the pumped current. In Fig. 5(a), we also see a second resonant point with much broader peak. Near this resonant level, we see that

TABLE I. Symmetry of the pumped currents on inversion of the magnetic field for both adiabatic and nonadiabatic electron pumps.

\begin{tabular}{|c|c|c|c|c|}
\hline \multirow[b]{2}{*}{ Symmetry } & \multicolumn{2}{|c|}{ Adiabatic pump } & \multicolumn{2}{|c|}{ Nonadiabatic pump } \\
\hline & $\phi_{12}=n \pi$ & $\phi_{12} \neq n \pi$ & $\phi_{12}=n \pi$ & $\phi_{12} \neq n \pi$ \\
\hline IUD & $I=0^{\mathrm{a}}$ & $I(B)=I(-B)^{\mathrm{a}}$ & $I(B)=I(-B)^{\mathrm{a}}$ & $I(B)=I(-B)^{\mathrm{a}}$ \\
\hline ILR & $I=0^{\mathrm{a}}$ & $I(B)=-I(-B)^{\mathrm{a}}$ & $I(B)=-I(-B)^{\mathrm{a}}$ & $I(B)=-I(-B)^{\mathrm{a}}$ \\
\hline IIV & $I=0^{\mathrm{a}}$ & $I=0^{\mathrm{a}}$ & $I=0^{\mathrm{a}}$ & $I=0^{\mathrm{a}}$ \\
\hline GUD & $I=0^{\mathrm{a}}$ & $I(B)=-I(-B)^{\mathrm{a}}$ & $I(B)=I(-B)^{\mathrm{a}}$ & $I(B, \phi)=I(-B,-\phi)^{\mathrm{a}}$ \\
\hline GLR & $I=0^{\mathrm{a}}$ & $I(B)=I(-B)^{\mathrm{a}}$ & $I(B)=-I(-B)^{\mathrm{a}}$ & $I(B, \phi)=-I(-B,-\phi)^{\mathrm{a}}$ \\
\hline GIV & $I=0^{\mathrm{a}}$ & $\begin{aligned} I & \approx 0^{\mathrm{b}} \\
I(B) & \approx I(-B)^{\mathrm{c}}\end{aligned}$ & $I=0^{\mathrm{a}}$ & $\begin{aligned} I(B, \phi) & =-I(B,-\phi)^{\mathrm{a}} \\
I(B) & \approx I(-B)^{\mathrm{c}}\end{aligned}$ \\
\hline
\end{tabular}

${ }^{a}$ Stands for the theoretical prediction from Ref. 29 confirmed by our numerical calculation.

${ }^{\mathrm{b}}$ Represents theoretical relation without numerical sustainment.

${ }^{\mathrm{c}}$ Corresponds to our new finding in contrast to the theoretical prediction. 

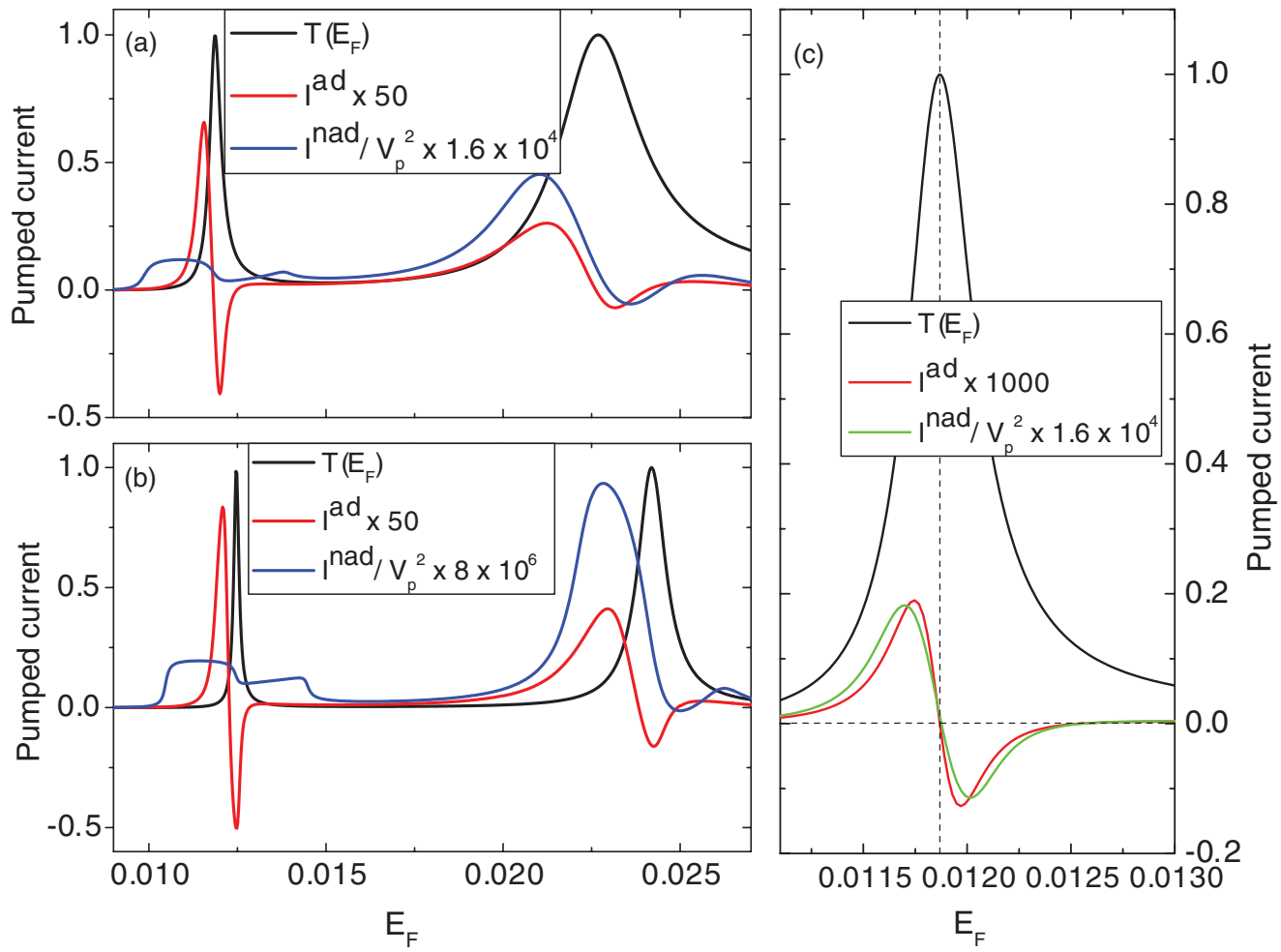

FIG. 5. (Color online) (a), (b): The pumped current as well as transmission coefficient as a function of Fermi energy at static potential height $V_{0}=1.0$ and 5.0, respectively. For visualization purposes, a factor is multiplied to the pumped current in Fig. 5. For $I^{\text {ad }}$, the factor is 50 in both (a) and (b). For $I^{\mathrm{nad}} / V_{p}^{2}$, this factor is $1.6 \times 10^{4}$ in (a) and $8 \times 10^{6}$ in (b). Other parameters: $B=0.001, \phi_{12}=\pi / 2 . V_{p}=0.5$, $\omega=0.002$ in (a) and $V_{p}=4.5, \omega=0.002$ in (b). (c) Highlights the pumped current at small pumping amplitudes at the first resonant peak; $V_{0}=1.0$ and $V_{p}=0.05, \omega=0.0002$. The factors for $I^{\mathrm{ad}}$ and $I^{\mathrm{nad}} / V_{p}^{2}$ are 1000 and $1.6 \times 10^{4}$, respectively.

the transmission coefficient and the pumped currents are well correlated. The resonant feature of the pumped current is also related to the width of the resonant peak in the transmission coefficient. Similar behaviors are found for a higher static potential barrier $V_{0}=5.0$ in Fig. 5(b). A larger barrier makes the resonant peaks much sharper, but it does not qualitatively affect the pumped current. The noticeable difference is that the pumped current peaks are shifted with that of the transmission coefficient. In addition, it seems that the nonadiabatic pumped current develops a plateau region near the first resonant peak.

When zooming in at the first resonant peak $\left[E_{F}=0.01187\right.$ in Fig. 5(a)], we found that at small pumping amplitude, the pumped currents are zeros for both adiabatic and nonadiabatic cases when a complete transmission occurs (transmission coefficient $T=1$ ). The numerical evidence is shown in Fig. 5(c). Note that there is only one transmission channel for the incident energy so that $T=1$ corresponds to complete transmission. We emphasize that the nonadiabatic pumped current goes to zero near the resonance only for very small frequency. At larger frequency, such as the case in Figs. 5(a) or 5(b), it is nonzero. For the nonadiabatic case, the pumped current at complete transmission is in general nonzero. However, if the frequency is very small, the adiabatic case is recovered. This is numerically supported by Fig. 5(c), where the two current curves are very similar.

Furthermore, the behavior of the pumped current for a structure exhibiting antiresonance phenomena was studied and the numerical results are shown in Fig. 6. To establish antiresonance, we use a $T$ junction, ${ }^{33}$ which is schematically plotted in the inset of Fig. 6. The side bar has longitudinal dimension 20 and transverse dimension 30. Pumping potentials are placed

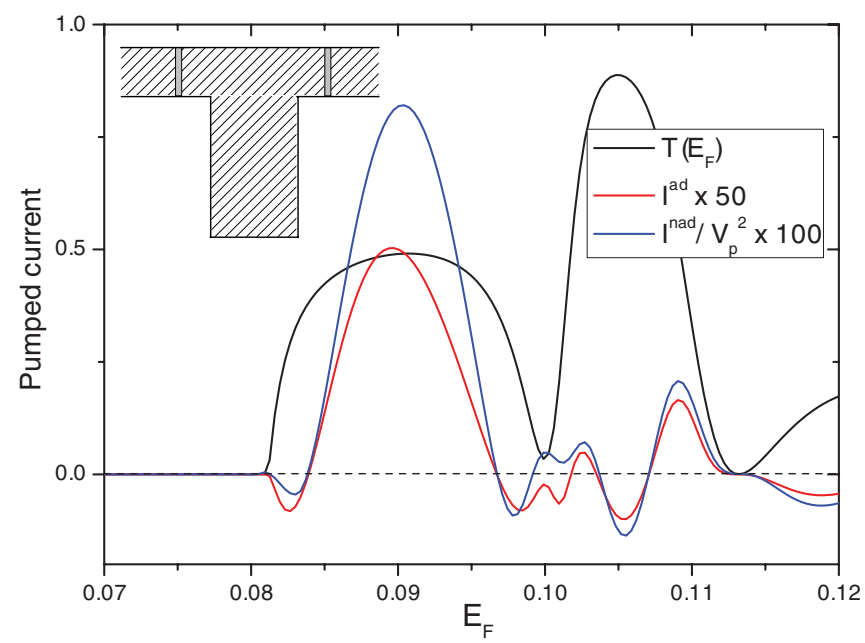

FIG. 6. (Color online) The pumped current versus Fermi energy in a $T$-shaped system. The side bar is of length 20 and width 30 . Two gray blocks indicate the positions where the pumping potentials are applied and the static potential is set to be zero. A factor of 50 is multiplied to $I^{\text {ad }}$ and it is 100 for $I^{\mathrm{nad}} / V_{p}^{2}$. Other parameters: $B=0.001, \phi_{12}=\pi / 2, V_{p}=0.05, \omega=0.002$. 


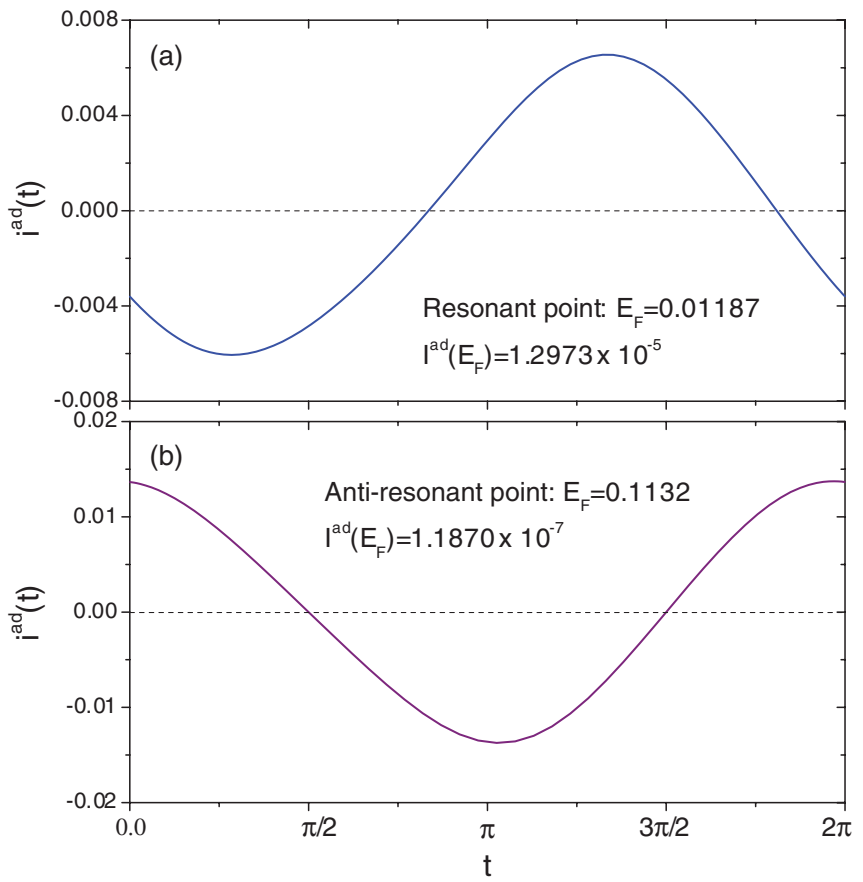

FIG. 7. (Color online) (a) Adiabatic pumped current in one pumping period at the resonant energy $E_{F}=0.01187 . V_{0}=1.0$ and $V_{p}=0.1$ in this panel. (b) Adiabatic pumped current in one pumping period at the antiresonant energy $E_{F}=0.1132 . V_{p}=0.05$ in this panel. Other common calculation parameters: $\phi_{12}=\pi / 2, B=0.001$.

on the two arms of the device and there are no static potential barriers in the system. From the transmission curve shown in Fig. 6, one clearly finds that $T\left(E_{F}\right)$ drops sharply to zero around $E_{F}=0.1132$, which is the signature of antiresonance. At this point, both the adiabatic and nonadiabatic pumped current are zero. Different from the resonant case, here, the range where the current is zero or nearly zero is much broader. We also see that at transmission minimum $E_{F}=0.10$ with small but nonzero transmission coefficient, the pumped current is nonzero, which indicates that the vanishing pumped current can only be observed at the antiresonance point.

Theoretically, it is difficult to show why the adiabatic pumped current vanishes at the resonant or antiresonant points. In Fig. 7, we provide a numerical explanation for the zero points of $I^{\text {ad }}$ at both resonance and antiresonance. Given $I^{\text {ad }}=\int_{0}^{2 \pi} i^{\text {ad }}(t) d t$, we see that the integrand $i^{\text {ad }}$ remains finite during the whole pumping period $t \in[0,2 \pi]$. But, the average current $I^{\text {ad }}$ is zero. This statement holds for both resonance and antiresonance cases. We note that, in both panels of Fig. 7, there is no clear symmetry of $i^{\text {ad }}(t)$ in the pumping period. Therefore, we are not able to give a theoretical explanation for the vanishing adiabatic pumped current at resonances.

In Figs. 8(a) and 8(b), we examine the influence of pumping amplitude $V_{p}$ or pumping frequency $\omega$ on the pumped currents with the static potential barrier fixed at $V_{0}=1$, which corresponds to the case shown in Fig. 5(a). At the first resonant energy $E_{F}=0.01187$, we plot $I^{\text {ad }}$ versus $V_{p}$, the pumping potential amplitude. The nonadiabatic pumped current $I^{\text {nad }}$ as a function of the pumping frequency $\omega$ is evaluated at the second resonant peak $E_{F}=0.0242$. In both cases, magnitudes
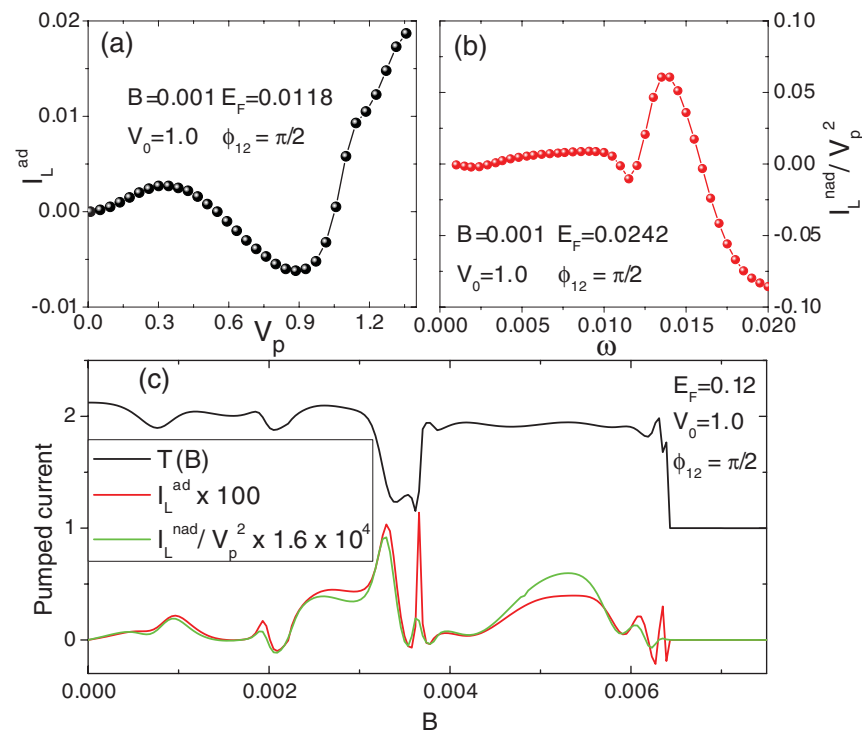

FIG. 8. (Color online) (a) Adiabatic current vs pumping potential $V_{p}$ at $E_{F}=0.0118$. (b) Nonadiabatic current versus pumping frequency $\omega$ at $E_{F}=0.0242$. System parameters: $\phi_{12}=\pi / 2, V_{0}=$ $1, B=0.001$. (c) Shows the pumped current and transmission coefficient versus magnetic field $B$ at Fermi energy $E_{F}=0.12$, with other parameters: $\phi_{12}=\pi / 2, V_{0}=1, V_{p}=0.5, \omega=0.002$. For illustration, a factor of 100 is multiplied to $I^{\text {ad }}$ and it is $1.6 \times 10^{4}$ for $I^{\mathrm{nad}} / V_{p}^{2}$.

of the pumped current changes in a oscillatory fashion with the increasing of $V_{p}$ or $\omega$. The pumped current can change its sign, which also reflects the nature of the parametric pump and manifest distinction between the pumped current and the conventional resonant tunneling current.

The resonance behavior of pumped current is also visible in Fig. 8(c), in which we depict $I_{p}$ and transmission coefficient versus magnetic field $B$ at Fermi energy $E_{F}=0.12$. The calculation is performed on a system with GLR symmetry, and the length of the pumping potential is 20 in the setup. Sweeping through magnetic field, there is a sharp change of transmission coefficient near $B \sim 0.003$ and the pumped current changes accordingly. With increasing magnetic field, $T$ becomes quantized (there is only one transmission channel at this magnetic field), indicating the occurrence of edge states in the quantum Hall regime, and the pumped current vanishes. In our setup, the electron pump operates by cycling modulation of electron passing through the pumping potentials, which are on top of static barriers defining the system. With increasing of the magnetic field, the electron wave function tends to localize near the edge, which decreases the modulation efficiency of the pumping potentials. As the edge state emerges, the electron will circumvent the confining potentials with no reflection during their deformations. In this case, the variation of the pumping potential has no effect on the moving electron. Hence, there is no pumped current when the edge state is formed in the system. Mathematically, it is also easy to show that when the instantaneous reflection coefficient vanishes (in the case of edge state) in the whole pumping period, there is no adiabatic pumped current in a two-probe system. The argument is shown below. 


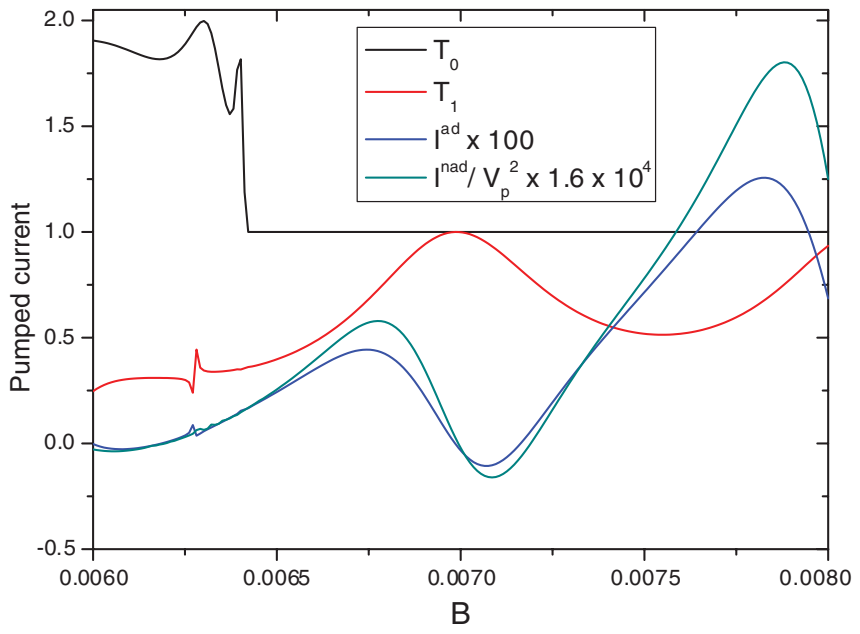

FIG. 9. (Color online) The pumped current as well as transmission coefficient as a function of magnetic field $B$ at Fermi energy $E_{F}=0.12$. Other parameters: $\phi_{12}=\pi / 2, V_{0}=1, V_{p}=0.5, \omega=$ 0.002 . $I^{\text {ad }}$ and $I^{\mathrm{nad}} / V_{p}^{2}$ are scaled by factors of 100 and $1.6 \times 10^{4}$, respectively.

For the case of the adiabatic pump, it is easy to understand why $I^{\text {ad }}$ is zero at $T=1$ (only one conducting channel in the system). For a perfect transmission, the diagonal terms $S_{L L}$ and $S_{R R}$ of the four-block scattering matrix are zero. Hence, we have $S_{L R}=S_{R L}=\exp (i \theta)$. From Eq. (3), we have

$$
d N_{\alpha} / d V_{j}=\left(i \partial \theta / \partial V_{j}\right) / \pi .
$$

For two pumping potentials, the current can be expressed in parameter space. Using the Green's theorem, Eq. (2) becomes ${ }^{5}$

$$
\begin{aligned}
I_{\alpha} & =\frac{1}{\tau} \int_{0}^{\tau} d t \frac{d Q_{\alpha}(t)}{d t} \\
& =\frac{q \omega}{2 \pi} \int d V_{1} d V_{2}\left(\frac{\partial}{\partial V_{1}} \frac{d N_{\alpha}}{d V_{2}}-\frac{\partial}{\partial V_{2}} \frac{d N_{\alpha}}{d V_{1}}\right) .
\end{aligned}
$$

From Eq. (8), it is easy to see that the integrand is zero. Hence, $I_{\alpha}=0$ if $S_{\alpha \alpha}=0$.

We also provide a numerical evidence for the above statement, which is shown in Fig. 9. In contrast to the calculation of Fig. 8(c), the static potential barriers extend to a width 40 , which is exactly the width of the scattering region. At the same time, the pumping barriers remain the same as before (with width 10). Now, the static transmission coefficient, labeled $T_{1}$ in the figure, does not have quantized value, but exhibits a resonant behavior. $T_{0}$ is copied from Fig. 8 for comparison. As long as the edge state of an electron is scattered with transmitted and reflected modes, the pumped current will be generated with varying system parameters.

\section{CONCLUSION}

In conclusion, we have studied the pumped current as a function of pumping potential, magnetic field, and pumping frequency in the resonant and antiresonant tunneling regimes. Resonant features are clearly observed for adiabatic and nonadiabatic pumped current. We found that when the resonant peak is sharp, the adiabatic pumped current changes sign near the resonance, while the nonadiabatic pumped current does not. When the resonant peak is broad, the behaviors of the pumped current in the adiabatic and nonadiabatic regimes are similar and both change sign near the resonance. At antiresonance, however, both the adiabatic and nonadiabatic pumped currents are zero. As the system enters the quantum Hall regime, the pumped currents vanish in all the setups shown in Fig. 1 since the pumping potentials can not modulate the electron wave function. Furthermore, we have numerically investigated the symmetry of the adiabatic and nonadiabatic pumped current of systems with different symmetries placed in magnetic field. The calculated results are listed in Table I and most of them are in agreement with the former theoretical results derived from the Floquet scattering matrix theory. Different from the theoretical prediction, we found that the system with general spatial inversion symmetry (GIV) gives rise to a finite pumped current at the adiabatic regime. At small magnetic field, both the adiabatic and nonadiabatic currents have an approximation relation $I(B) \approx I(-B)$.

\section{ACKNOWLEDGMENTS}

This work was supported by RGC (Grant No. HKU 705409P), University Grant Council (Contract No. AoE/P04/08) of the Government of HKSAR, and a CRCG grant from the University of Hong Kong. Y.X. Xing is also supported by the NSF-China under Grant No. 11174032. The computational work was partially performed on HPCPOWER2 system of the computer center, The University of Hong Kong. *jianwang@hku.hk

${ }^{1}$ D. J. Thouless, Phys. Rev. B 27, 6083 (1983).

${ }^{2}$ Q. Niu and D. J. Thouless, J. Phys. A: Math. Gen. 17, 2453 (1984).

${ }^{3}$ Q. Niu, Phys. Rev. Lett. 64, 1812 (1990).

${ }^{4}$ F. Hekking and Yu. V. Nazarov, Phys. Rev. B 44, 9110 (1991).

${ }^{5}$ P. W. Brouwer, Phys. Rev. B 58, R10135 (1998).

${ }^{6}$ I. L. Aleiner and A. V. Andreev, Phys. Rev. Lett. 81, 1286 (1998).

${ }^{7}$ T. A. Shutenko, I. L. Aleiner, and B. L. Altshuler, Phys. Rev. B 61, 10366 (2000).

${ }^{8}$ Y. D. Wei, J. Wang, and H. Guo, Phys. Rev. B 62, 9947 (2000).

${ }^{9}$ M. Moskalets and M. Büttiker, Phys. Rev. B 66, 205320 (2002).
${ }^{10}$ S. W. Chung, C. S. Tang, C. S. Chu, and C. Y. Chang, Phys. Rev. B 70, 085315 (2004).

${ }^{11}$ M. Switkes, C. M. Marcus, K. Campman, and A. C. Gossard, Science 283, 1907 (1999).

${ }^{12}$ Y. D. Wei, J. Wang, H. Guo, and C. Roland, Phys. Rev. B 64, 115321 (2001).

${ }^{13}$ V. I. Talyanskii, D. S. Novikov, B. D. Simons, and L. S. Levitov, Phys. Rev. Lett. 87, 276802 (2001).

${ }^{14}$ R. Zhu and H. Chen, Appl. Phys. Lett. 95, 122111 (2009).

${ }^{15}$ Q. Zhang, K. S. Chan, and Z. Lin, Appl. Phys. Lett. 98, 032106 (2011). 
${ }^{16}$ S. K. Watson, R. M. Potok, C. M. Marcus, and V. Umansky, Phys. Rev. Lett. 91, 258301 (2003).

${ }^{17}$ A. G. Malshukov, C. S. Tang, C. S. Chu, and K. A. Chao, Phys. Rev. B 68, 233307 (2003).

${ }^{18}$ R. Benjamin and C. Benjamin, Phys. Rev. B 69, 085318 (2004).

${ }^{19}$ M. Swikes, Ph.D. thesis, Stanford University, 1999.

${ }^{20}$ P. W. Brouwer, Phys. Rev. B 63, 121303 (2001).

${ }^{21}$ A. Andreev and A. Kamenev, Phys. Rev. Lett. 85, 1294 (2000).

${ }^{22}$ S. W. Kim, Phys. Rev. B 66, 235304 (2002).

${ }^{23}$ B. G. Wang, J. Wang, and H. Guo, Phys. Rev. B 65, 073306 (2002).

${ }^{24}$ B. G. Wang, J. Wang, and H. Guo, Phys. Rev. B 68, 155326 (2003).

${ }^{25}$ O. Entin-Wohlman and A. Aharony, Phys. Rev. B 66, 035329 (2002).
${ }^{26}$ A. Agarwal and D. Sen, J. Phys.: Condens. Matter 19, 046205 (2007).

${ }^{27}$ F. Zhou, B. Spivak, and B. L. Altshuler, Phys. Rev. Lett. 82, 608 (1999).

${ }^{28}$ I. L. Aleiner, B. L. Altshuler, and A. Kamenev, Phys. Rev. B 62, 10373 (2000).

${ }^{29}$ S. W. Kim, Phys. Rev. B 68, 085312 (2003).

${ }^{30}$ M. Moskalets and M. Büttiker, Phys. Rev. B 72, 035324 (2005).

${ }^{31}$ C. S. Li, Y. J. Yu, Y. D. Wei, and J. Wang, Phys. Rev. B 75, 035312 (2007).

${ }^{32}$ M. Büttiker, J. Phys.: Condens. Matter 5, 9361 (1993); M. Büttiker, H. Thomas, and A. Prêtre, Z. Phys. B 94, 133 (1994).

${ }^{33}$ F. Sols, M. Macucci, U. Ravaioli, and K. Hess, Appl. Phys. Lett. 54, 350 (1989). 